CARDIOVASCULAR MEDICINE

\title{
Limited long term effects of a management programme for heart failure
}

\author{
M Mejhert, T Kahan, H Persson, M Edner
}

See end of article for authors' affiliations

....................

Correspondence to: Dr M Mejhert, Karolinska Institutet Danderyd Hospital, Section of Cardiology, S-182 88 Stockholm, Sweden; marit.mejhert@ med.ds.sll.se

Accepted

26 September 2003
Heart 2004;90:1010-1015. doi: 10.1136/hrt.2003.014407

Objective: To evaluate the effects of a nurse based outpatient management programme for elderly patients discharged with heart failure from a university hospital.

Design: Patients with heart failure (New York Heart Association class II-IV) and left ventricular systolic dysfunction aged 60 years or more were randomly assigned to follow up within the management programme or to conventional follow up, usually in primary care. Of the 208 participants, $58 \%$ were men, mean age was 75 years, and mean ejection fraction 34\%. All patients were scheduled for three observational study visits at six month intervals. The primary end point was quality of life (QoL) and secondary end points were hospitalisation and mortality.

Results: More patients achieved target doses of angiotensin converting enzyme (ACE) inhibitors in the intervention group than in the control group $(82 \% \vee 69 \%, 88 \% \vee 69 \%$, and $88 \%$ v $74 \%$ of recommended target doses at 6,12, and 18 months of follow up, respectively, $p<0.05$ for all). Patients with initial low QoL had a poor prognosis. After a mean 1122 days of follow up, $82 \%$ of all patients had been readmitted. There were on average 4.7 readmissions per patient and $66 \%$ were due to non-cardiac diagnoses. There were no differences in QoL or health care consumption between the two study groups during follow up. Conclusion: A nurse based management programme is more effective than follow up in primary care in optimising medication for elderly patients with heart failure. However, such a programme does not seem to have a favourable influence on QoL or readmission rate during long term follow up.
$\mathrm{T}$ he heart failure syndrome is characterised by poor quality of life (QoL), high hospitalisation rates, and poor prognosis. ${ }^{1-7}$ To improve QoL and clinical outcome, specialised management programmes for patients with heart failure have been advocated. The aims of these programmes are to educate and counsel patients about heart failure and to optimise medical treatment. Thus, the management programmes try to enhance self care behaviour in heart failurefor example, by teaching the patients to monitor deterioration of heart failure symptoms and to use a flexible diuretic regimen. Several large clinical trials on heart failure have shown improved QoL with pharmacological treatment and most management programmes aim at providing medication in consensus with guidelines. However, the management programmes use different organisation models and working strategies, which make data difficult to compare and to interpret. Also, the multifactorial design makes it unclear as to which intervention actually causes improvement. Measuring QoL and attributing causation is difficult. Furthermore, interpretation of data from different trials is complex, as a variety of questionnaires have been used. These and other limitations of QoL measurements have been reviewed elsewhere. $^{8}$

The long term effects of heart failure management programmes remain unclear, as most studies have short follow up times of less than one year. Recently, McAlister and colleagues ${ }^{9}$ in a systematic review compiled data from 11 prospective randomised trials, mostly of hospitalised patients with heart failure. ${ }^{9}$ They found a reduction in health care consumption (number of readmissions, lengths of stay, and total days of hospitalisation), although data on QoL and mortality were inconclusive. Thus, the aim of the present study was to evaluate the effect of a hospital located, nurse based heart failure management programme on QoL as compared with standard care, usually by follow up in primary care. The second aim was to evaluate hospitalisations and mortality during long term follow up.

\section{METHODS}

\section{Study design and population}

OPTIMAL (optimising congestive heart failure outpatient clinic project) is a randomised prospective study of patients hospitalised with heart failure between January 1996 and December 1999 in our institution. Danderyd University Hospital is a secondary referral centre situated in northeastern greater Stockholm with a catchment area by the time of the study of about 300000 inhabitants. In this region, characterised by a high socioeconomic standard, the population is somewhat older but healthier than the average Swede. The primary end point of the study was to assess whether a nurse monitored management programme at the hospital outpatient clinic would lead to improved QoL. Cardiac function, medication, hospitalisation, and mortality were secondary end points. All patients 60 years of age or older with heart failure according to New York Heart Association (NYHA) class II-IV and left ventricular systolic dysfunction by echocardiography were eligible for inclusion. Exclusion criteria were an acute myocardial infarction or unstable angina pectoris within the previous three months, valvar stenosis, dementia, severe concomitant disease, or refusal to participate. In all, 285 patients were screened for participation in the study. Of those, 77 patients were not included, as they did not wish to participate $(\mathrm{n}=32)$, did not have systolic dysfunction at the screening echocardiography $(\mathrm{n}=23)$, or met other exclusion criteria such as valvar

Abbreviations: $A C E$, angiotensin converting enzyme; NYHA, New York Heart Association; QoL, quality of life; OPTIMAL, optimising congestive heart failure outpatient clinic project 
stenosis $(\mathrm{n}=5)$, confusion $(\mathrm{n}=5)$, stroke or myocardial infarction $(n=6)$, or severe concomitant disease $(n=6)$. Thus, after informed consent, 208 patients were enrolled and underwent random assignment to follow up within the management programme (intervention group) or to conventional follow up, usually in primary care (control group). The study was approved by the ethics committee and complied with the Declaration of Helsinki.

\section{Management programme and intervention}

At Danderyd University Hospital, a nurse monitored management programme for patients with heart failure was set up in 1992. A senior cardiologist supervises the programme. After referral to the programme, the patient pays regular visits to the outpatient clinic and is encouraged to keep in contact with the nurse. At each visit the nurse checks symptoms and signs of heart failure, blood pressure, heart rate, and weight. Laboratory tests measure creatinine, sodium, and potassium concentrations. Before starting $\beta$ adrenoceptor blocking treatment an ECG is checked. Nurses working in the programme are allowed to institute and change the doses of angiotensin converting enzyme (ACE) inhibitors, angiotensin II receptor blockers, $\beta$ adrenoceptor blockers, potassium substitution, diuretics, and potassium sparing diuretics according to a standard protocol. The target dose of captopril is $100 \mathrm{mg} /$ day, enalapril $20 \mathrm{mg} /$ day, and ramipril $10 \mathrm{mg} /$ day. A routine for titration of $\beta$ adrenoceptor blockers was not set up until some years after the start of the study. The patient is instructed to check his or her weight regularly and to monitor early signs of deterioration. Patients with good compliance are instructed to change dosing of diuretics on their own. Dietary advice recommends restricted sodium, fluid, and alcohol intake. Information is repeated in booklets and computerised educational programmes.

According to our routine, written information is given in a structured format to the general practitioner at discharge of a patient from our institution. Patients randomised to the control group typically were followed up by their general practitioners to be treated according to the local health care plan for heart failure. There are 28 community health centres in the catchment area of our hospital. In cooperation with primary care practitioners, a health care plan for patients with heart failure was instituted in 1994. This states, for example, that an echocardiogram should be carried out for all patients with a history of an acute myocardial infarction or with clinical signs of heart failure. If left ventricular systolic function is reduced, with an ejection fraction $<40 \%$, an ACE inhibitor should be initiated. Table 1 outlines the health care plan for heart failure. Several meetings with hospital specialists and general practitioners have addressed the importance of optimised medication and education of patients with heart failure. A secondary prevention health

Table 1 Established health care programme for patients with heart failure in primary care and in hospital based outpatient care

Initial evaluation
History and physical examination
Electrocardiography
Echocardiography
Exercise testing
Chest radiography
Laboratory tests of haemoglobin, glucose, electrolytes, thyroid function
Treatment
Patient education according to a checklist
Drug treatment with diuretics, ACE inhibitors, spironolactone,
$\beta$ adrenoblockers
Surgical intervention or pacemaker treatment if needed

$\mathrm{ACE}$, angiotensin converting enzyme. care plan for patients with coronary heart disease is well established in a similar way. ${ }^{10}$

In addition to the visits outlined above, all patients were scheduled for clinical examinations and detailed control of medication at 6, 12, and 18 months at the Cardiovascular Research Laboratory in our institution. One and the same cardiologist met the patients at all visits. The patients were informed at the start of the study that these visits were purely observational and with no intention to initiate examinations or to change medication.

\section{Quality of life}

The Nottingham health profile is a non-disease specific questionnaire consisting of two parts. It has been tested for validity and reliability in the UK and in Sweden. ${ }^{11-13}$ Part 1 comprises 38 yes/no questions concerning emotional reaction, sleep, energy, pain, physical mobility, and social isolation. The answers are weighted and scores for the six dimensions and the total sum are calculated. The higher the score, with a maximum of 100 points in each dimension, the worse the QoL. Part 2 concerns activities of daily life and will be evaluated and reported on later. Reference values, originally derived from an English population, have been validated for use in Sweden. ${ }^{14}$ All patients in the study were asked to complete the questionnaire at baseline and at the three follow up visits under the supervision of a nurse. If a patient was unable to visit the hospital, the Nottingham health profile questionnaire was sent by mail.

\section{Hospitalisation and mortality}

Outcome on all cause mortality was determined from death certificates. Data on hospitalisation rates were collected from the National Board of Health and Welfare, where all hospital admissions in the catchment area of Stockholm are registered, as well as diagnosis, length of stay, and outpatient visits. By using coded versions of the patient's identity number, it was possible to obtain data on all health care consumption of each participant. We analysed admissions and outpatient visits during at least 18 months from inclusion and during the total study period, from January 1996 to June 2000. Cardiac causes were separated by use of the diagnosis related groups system.

\section{Echocardiography}

Echocardiography was performed with an Acuson $128 \mathrm{XP} / 10$ (Mountain View, California, USA). The ejection fraction was calculated according to the recommendations of the American Society of Echocardiography. ${ }^{15}$ Left ventricular systolic function was also evaluated by the atrioventricular plane displacement method. ${ }^{16}$ The atrioventricular plane displacement was measured by $\mathrm{M}$ mode from an apical window. Mean values from four sites and three beats or more were used and the overall mean was calculated. Patients were enrolled if a screening echocardiographic examination after medical stabilisation showed an ejection fraction $\leqslant 45 \%$ or atrioventricular plane displacement was $\leqslant 10 \mathrm{~mm}$. The screening measurements were not used further in the study and all echocardiographic findings presented in this paper are from an extensive baseline investigation, sometimes performed a few days after screening.

\section{Blood samples}

Blood chemistry was analysed by standard methods at baseline and at 12 months.

Blood samples for neurohormone measurements were obtained on ice after 30 minutes of supine rest and immediately centrifuged at $4^{\circ} \mathrm{C}$. Plasma was then frozen at $-70^{\circ} \mathrm{C}$ until analysis. Brain natriuretic peptide was measured 
Table 2 Clinical characteristics at baseline

\begin{tabular}{lllll}
\hline & $\begin{array}{l}\text { Total } \\
(\mathbf{n = 2 0 8 )}\end{array}$ & $\begin{array}{l}\text { Intervention } \\
\text { group }(\mathbf{n = 1 0 3 )}\end{array}$ & $\begin{array}{l}\text { Control group } \\
(\mathbf{n}=105)\end{array}$ & p Value \\
\hline Age (years) & $75.8(7.1)$ & $75.9(7.7)$ & $75.7(6.6)$ & NS \\
Male sex & $120(58 \%)$ & $58(56 \%)$ & $62(59 \%)$ & NS \\
Marital status single & $93(52 \%)$ & $46(52 \%)$ & $47(52 \%)$ & NS \\
NYHA class I/II/III/IV & $0 / 129 / 77 / 2$ & $0 / 60 / 43 / 0$ & $0 / 69 / 34 / 2$ & NS \\
Ejection fraction (\%) & $0.34(0.11)$ & $0.34(0.12)$ & $0.35(0.11)$ & NS \\
Systolic blood pressure (mm Hg) & $134(25)$ & $135(24)$ & $133(26)$ & NS \\
Diastolic blood pressure (mm Hg) & $79(14)$ & $79(14)$ & $79(13)$ & NS \\
Plasma BNP (pmol/I) & $331(36) 9$ & $342(323)$ & $320(412)$ & NS \\
Serum creatinine ( $\mu$ mol/I) & $115(37)$ & $114(36)$ & $117(39)$ & NS \\
Medical history & $119(57 \%)$ & $59(57 \%)$ & $60(57 \%)$ & NS \\
$\quad$ Previously known heart failure & $139(67 \%)$ & $65(63 \%)$ & $74(70 \%)$ & NS \\
Ischaemic heart disease & $111(53 \%)$ & $54(52 \%)$ & $57(54 \%)$ & NS \\
Arrhythmia & $65(31 \%)$ & $38(37 \%)$ & $27(26 \%)$ & NS \\
Hypertension & $46(22 \%)$ & $26(25 \%)$ & $20(19 \%)$ & NS \\
Diabetes mellitus & $20(10 \%)$ & $10(10 \%)$ & $10(10 \%)$ & NS \\
Cardiomyopathy & $17(8 \%)$ & $9(8 \%)$ & $8(8 \%)$ & NS \\
Valve disease & & & & \\
\hline Data are mean (SD). & & & & \\
BNP, brain natriuretic peptide; NS, not significant; NYHA, New York Heart Association. & \\
\hline
\end{tabular}

by an immunoradiometric assay (Shionoria Cis Bio International, Osaka, Japan) at baseline and at 12 months. ${ }^{17}$

\section{Statistical analysis}

To detect a clinically significant difference in QoL at 18 months of follow up (more than four points for each dimension) with $\beta=0.80$ and $2 \alpha=0.05$ it was calculated that 180 evaluable patients would be required. To account for some $10-15 \%$ of patients estimated to be lost during the course of the study we intended to enrol 210 participants. Data are presented as mean (SD). Analyses were conducted according to the intention to treat principle. If the rate of missing answers in one dimension

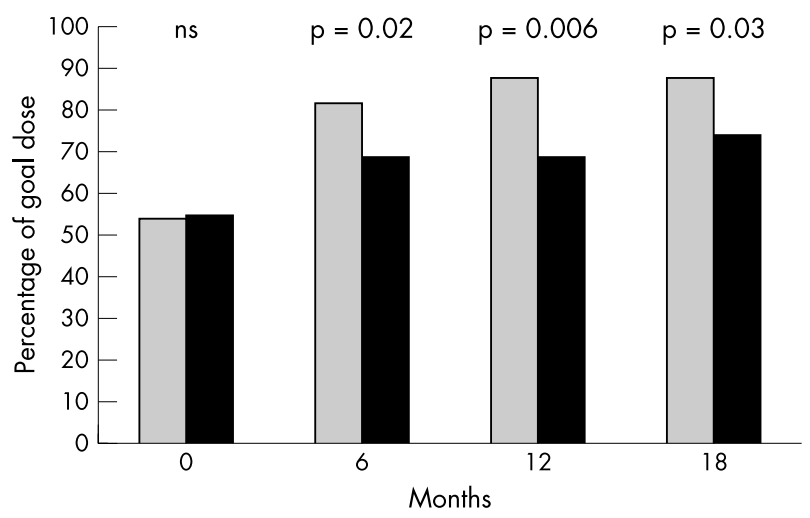

Figure 1 Achieved percentage of target dose of angiotensin converting enzyme inhibitors during 18 months of follow up. Grey bars, intervention group ( $\mathrm{n}=103$ ); black bars, control group ( $\mathrm{n}=105)$. was $<30 \%$, the missing value was substituted by the mean value of the entire group. The last follow up value during the 18 month period was carried over for patients alive but with missing data. Unpaired data were evaluated with Student's independent $t$ test. A two way repeated measures analysis of variance was used to evaluate changes over time. Survival curves were generated by the KaplanMeier method and log rank tests were used to assess differences in survival. A probability value of $p<0.05$ was considered significant.

\section{RESULTS}

\section{Baseline characteristics}

Table 2 shows clinical characteristics at baseline. Women were older than the men ( $78 v 74$ years, $\mathrm{p}=0.009)$. The two study groups were comparable and the distribution of NYHA class did not differ at baseline.

All patients were followed up for at least 18 months. The mean follow up was 1122 (405) days. Patients in the intervention group made a mean (SD) of 2.2 (2.3) (median 1, range $0-10$ ) visits to the nurse management programme.

\section{Effects on medications and on blood chemistry}

As fig 1 shows, more patients achieved goal doses of ACE inhibitors in the intervention group than in the control group at all three follow up visits. There were no other differences in medication within or between the two groups during follow up (table 3). Not all patients were treated with ACE inhibitors because of symptomatic hypotension or renal dysfunction. $\beta$ Adrenoblocker dosage was not chosen as a treatment end point because routine treatment with $\beta$ adrenoblockers was

Table 3 Proportions of patients taking medication at baseline and during follow up

\begin{tabular}{|c|c|c|c|c|c|c|c|c|}
\hline & \multicolumn{4}{|c|}{ Intervention group } & \multicolumn{4}{|c|}{ Control group } \\
\hline & Baseline & 6 months & 12 months & 18 months & Baseline & 6 months & 12 months & 18 months \\
\hline Number & 103 & 67 & 56 & 50 & 105 & 83 & 74 & 66 \\
\hline Mean furosemide dose (mg/day) & 114 & 92 & 77 & 81 & 120 & 106 & 106 & 89 \\
\hline Furosemide (\%) & 94 & 93 & 91 & 92 & 92 & 95 & 95 & 87 \\
\hline$\beta$ Adrenoblockers (\%) & 52 & 57 & 55 & 57 & 52 & 58 & 62 & 63 \\
\hline ACE inhibitors (\%) & 72 & 76 & 66 & 63 & 76 & 80 & 77 & 68 \\
\hline Digitalis (\%) & 54 & 49 & 45 & 40 & 48 & 48 & 36 & 40 \\
\hline Aldosterone antagonists (\%) & 21 & 18 & 14 & 16 & 18 & 18 & 15 & 18 \\
\hline
\end{tabular}


Table 4 Quality of life at baseline according to age group and sex in the study group (Study) and a reference group ${ }^{17}$ (Ref)

\begin{tabular}{|c|c|c|c|c|c|c|c|c|}
\hline & \multicolumn{2}{|c|}{$60-64$ years } & \multicolumn{2}{|c|}{$65-69$ years } & \multicolumn{2}{|c|}{$70-74$ years } & \multicolumn{2}{|c|}{$>75$ years } \\
\hline & Study & Ref & Study & Ref & Study & Ref & Study & Ref \\
\hline Total (M/F) & $181 / 77$ & $69 / 73$ & $124 / 119$ & $58 / 119$ & $125 / 201$ & $67 / 188$ & $146 / 181$ & $118 / 165$ \\
\hline Emotional reaction (M/F) & $32 / 12$ & $16 / 19$ & $11 / 8$ & $14 / 24$ & $14 / 30$ & $20 / 34$ & $16 / 21$ & $29 / 44$ \\
\hline Sleep (M/F) & $35 / 22$ & $8 / 10$ & $33 / 33$ & $9 / 21$ & $31 / 44$ & $7 / 16$ & $27 / 36$ & $14 / 26$ \\
\hline Energy $(\mathrm{M} / \mathrm{F})$ & $52 / 30$ & $10 / 10$ & $40 / 40$ & $5 / 13$ & $46 / 61$ & $9 / 15$ & $52 / 59$ & $13 / 17$ \\
\hline Pain $(M / F)$ & $17 / 5$ & $20 / 20$ & $15 / 10$ & $20 / 39$ & $7 / 17$ & $17 / 30$ & $13 / 18$ & $31 / 30$ \\
\hline Physical mobility (M/F) & $27 / 8$ & $7 / 6$ & $22 / 21$ & $3 / 6$ & $22 / 35$ & $4 / 11$ & $29 / 34$ & $10 . / 12$ \\
\hline Social isolation (M/F) & $19 / 0$ & $8 / 9$ & $3 / 6$ & $7 / 18$ & $5 / 14$ & $10 / 17$ & $10 / 13$ & $21 / 36$ \\
\hline
\end{tabular}

$F$, female; $M$, male.

Data are means.

\begin{tabular}{|c|c|c|}
\hline & $\begin{array}{l}\text { Intervention } \\
\text { group }(n=103)\end{array}$ & $\begin{array}{l}\text { Control group } \\
(n=105)\end{array}$ \\
\hline \multicolumn{3}{|l|}{ Baseline } \\
\hline Total & $162(115)$ & 150 (119) \\
\hline Emotional reaction & $20(23)$ & $18(23)$ \\
\hline Sleep & $33(31)$ & $32(33)$ \\
\hline Energy & $53(38)$ & $51(40)$ \\
\hline Pain & $16(21)$ & $12(20))$ \\
\hline Physical mobility & $30(26)$ & $27(26)$ \\
\hline Social isolation & $10(1)$ & 10 (17) \\
\hline \multicolumn{3}{|l|}{6 months } \\
\hline Total & $132(100)$ & 117 (107) \\
\hline Emotional reaction & $13(18)$ & 12 (19) \\
\hline Sleep & $25(26)$ & $24(27)$ \\
\hline Energy & $41(38)$ & $37(37)$ \\
\hline Pain & $18(22)$ & $11(18)$ \\
\hline Physical mobility & $24(23)$ & $21(22)$ \\
\hline Social isolation & $10(20)$ & $11(19)$ \\
\hline \multicolumn{3}{|l|}{12 months } \\
\hline Total & $136(107)$ & 127 (117) \\
\hline Emotional reaction & 14 (19) & $15(23)$ \\
\hline Sleep & $23(24)$ & $27(28)$ \\
\hline Energy & $46(38)$ & $38(41)$ \\
\hline Pain & $15(24)$ & $12(18)$ \\
\hline Physical mobility & $27(24)$ & $23(18)$ \\
\hline Social isolation & 11 (19) & $12(19)$ \\
\hline \multicolumn{3}{|l|}{18 months } \\
\hline Total & $134(11)$ & $130(125)$ \\
\hline Emotional reaction & $14(21)$ & $15(23)$ \\
\hline Sleep & $23(25)$ & $26(31)$ \\
\hline Energy & $44(40)$ & $42(42)$ \\
\hline Pain & $14(22)$ & $11(18)$ \\
\hline Physical mobility & $27(25)$ & $26(27)$ \\
\hline Social isolation & $11(20)$ & $11(20)$ \\
\hline
\end{tabular}

implemented in the management programme during the study. Routine blood chemical analysis (concentrations of creatinine, sodium, and potassium in serum, and glucose in plasma) and brain natriuretic peptide did not differ between the study groups at baseline. Values were unchanged at 12 months (data not shown).
Table 6 Total quality of life scores and survival

\begin{tabular}{llll}
\hline & Survivors & Non-survivors & p Value \\
\hline Baseline & $142(115)$ & $182(117)$ & 0.029 \\
6 months & $112(98)$ & $165(113)$ & 0.008 \\
12 months & $117(106)$ & $180(121)$ & 0.004 \\
18 months & $113(111)$ & $195(121)$ & $<0.001$ \\
\hline \multicolumn{2}{l}{ Data are mean (SD). A high score indicates a poor quality of life. }
\end{tabular}

\section{Quality of life}

Compared with a reference population, mean total scores of QoL were higher in the study population at baseline, indicating a worse QoL (table 4). ${ }^{18}$ In both sexes and in all age groups the variables sleep, energy, and physical mobility were affected more in the study population than in the reference population (table 5).

Women reported higher total scores than men, both at baseline and during follow up $(\mathrm{p}=0.022)$. When the study population was subdivided by median age (76 years), the group of elderly patients (mean 81 years) did not differ in total scores from the younger group (mean 70 years) at baseline, but total scores at follow up were higher in the elderly $(p=0.018)$. In the entire study population, QoL improved from baseline to 18 months $(p=0.032)$ with no differences between the intervention group and the control group (table 5 ).

Baseline QoL was reduced in patients who subsequently died $(\mathrm{n}=74)$ compared with survivors $(\mathrm{n}=134)$ (table 6). The high QoL scores were attributed to all six dimensions except pain (data not shown).

\section{Readmissions and mortality}

As table 7 shows, the readmissions were for both cardiac and non-cardiac diagnoses. In all, 28676 outpatient visits were made-that is, a mean of 4.7 visits per patient and month. Visits were for both hospital care and primary care and for all kinds of caretakers.

During the first 18 months after inclusion 49 patients died after a mean (SD) of 188 (143) days. During the entire follow

Table 7 Mortality and numbers of readmissions during follow up

\begin{tabular}{|c|c|c|c|c|}
\hline & $\begin{array}{l}\text { Total } \\
(n=208)\end{array}$ & $\begin{array}{l}\text { Intervention } \\
\text { group }(n=103)\end{array}$ & $\begin{array}{l}\text { Control group } \\
(n=105)\end{array}$ & p Value \\
\hline All cause mortality & 74 & 40 & 34 & NS \\
\hline Patients readmitted within 18 months & 138 & 69 & 69 & NS \\
\hline Patients readmitted within follow up & 171 & 85 & 86 & NS \\
\hline Readmissions/patient during follow up & 4.7 & 4.4 & 4.9 & NS \\
\hline Time to first readmission, all cause (days) & 270 & 246 & 294 & NS \\
\hline Length of stay, first readmission, all cause (days) & 3.9 & 3.7 & 4.1 & NS \\
\hline
\end{tabular}



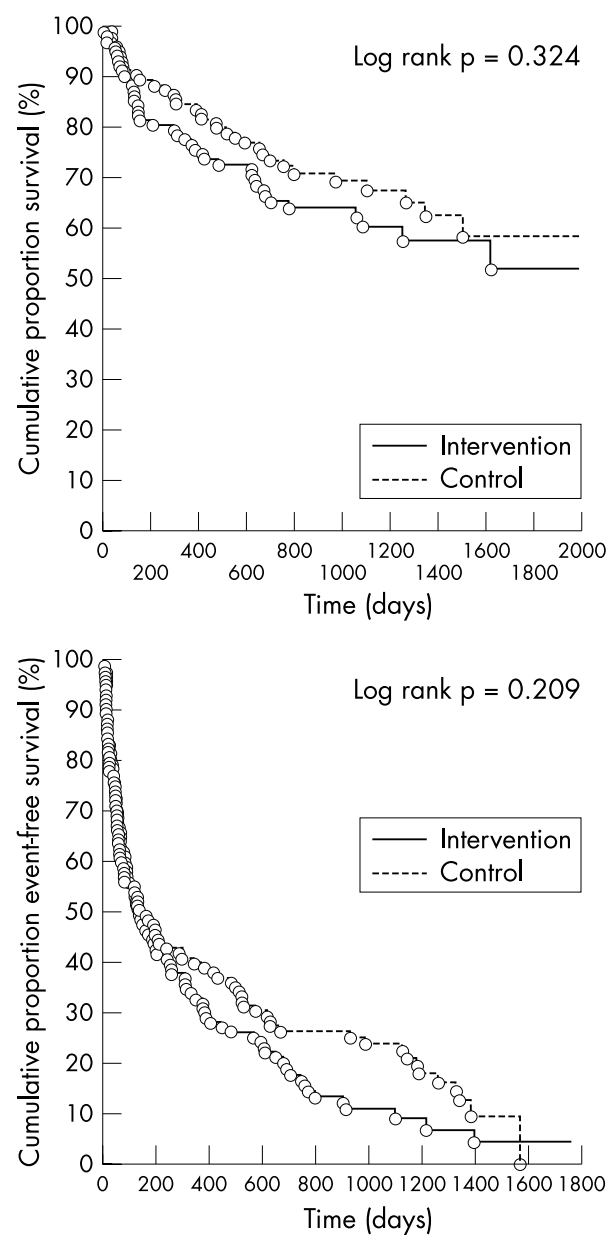

Figure 2 Survival and the combined end point of readmission and death during follow up.

up, 74 patients died after 423 (391) days. Figure 2 shows survival curves and the combined end point of readmission and death in the two study groups. Only $12 \%$ of all study patients had event-free survival. We found no difference in health care consumption or mortality between the two study groups.

\section{DISCUSSION}

\section{Drug treatment}

Compared with the control group, patients in the intervention group of the present study achieved higher doses of ACE inhibitors. In this group of elderly patients with heart failure, $90 \%$ of those treated with ACE inhibitors achieved the target dose. This may be important, as higher doses of ACE inhibitors improve the prognosis for patients with heart failure, ${ }^{19}$ although this was not an objective of our study. We have previously shown that patients in the intervention group improved their self care behaviour, measured as knowledge of how to monitor deterioration and how to use a flexible diuretic regimen. ${ }^{20}$ Thus, our results show that the current nurse based management programme is well suited for older patients with heart failure and favourably affects these patients. Of note, a high proportion (55\%) of the study patients already had optimal doses of ACE inhibitors at baseline.

Earlier studies on management programmes have reported a low use of heart failure medication. Of patients enrolled from 1991 to 1993 in a Swedish study only 23\% received ACE inhibitors and 10\% $\beta$ adrenoceptor blockers. ${ }^{21}$ In a 1995 study, Rich and colleagues ${ }^{22}$ reported that $54-64 \%$ and $11-$ $13 \%$ of patients were prescribed ACE inhibitors and $\beta$ adrenoceptor blockers, respectively. ${ }^{22}$ Indeed, in Sweden, a prescription rate of $20-25 \%$ of ACE inhibitors for ambulatory patients with heart failure has been reported throughout the past decade. ${ }^{23}$ However, two studies from Stockholm found a $50-60 \%$ prescription rate of ACE inhibitors for ambulatory patients with coronary heart diseas $\mathrm{e}^{10}$ and heart failure and for hospitalised patients with heart failure. ${ }^{24}$ In the study by Kahan and colleagues, ${ }^{10}$ the use of ACE inhibitors for heart failure was higher in the group followed up by the hospital specialists than in patients followed up by general practitioners $(70 \% \vee 55 \%)$. The use of $\beta$ adrenoceptor blockers, diuretics, and digoxin were similar in the hospital outpatient clinic and the primary care group. These results suggest that many patients with heart failure in our catchment area are being treated in accordance with current guidelines. Thus, the potential that improved ACE inhibitor use in the intervention group would transform into improved QoL and affect readmission rates or mortality in our study may have been limited. The good compliance with treatment guidelines in primary care may be the result of the health care programme for heart failure and coronary heart disease developed in our region for many years. ${ }^{10}$

\section{Quality of life}

The present study had a long follow up period, with an average of more than three years. Although the study showed that the management programme had favourable effects on optimising doses of ACE inhibitors and on patients' knowledge of how to monitor and prevent deterioration, the results failed to show an improvement in QoL. This supports findings by others. ${ }^{25}$ Some randomised studies on management programmes have shown that intervention improves QoL, whereas others have reported equivocal results. ${ }^{21} 22$ 26-28 Several reasons may contribute to these diverging results. Different management programmes have been used. The studies have been small. The follow up has often been limited in time. Several QoL questionnaires with important differences have been used. ${ }^{29}$ Taken together, the improvement in QoL by a nurse based management programme appears to be limited in an older general heart failure population, provided a health care programme for heart failure has already been implemented. The questionnaire used to evaluate QoL in the present study is well established and validated. ${ }^{11}$ However, the lack of effect may to some extent also be explained by the use of this non-disease specific QoL instrument. ${ }^{8}$ Also, the separate effects of the different interventions of the management programme have not been fully identified. Thus, the results must be interpreted carefully.

Interestingly, non-survivors already reported significantly worse QoL at baseline and this difference became more pronounced during follow up. This may suggest that a single evaluation of QoL of patients with heart failure differentiates patients with a poor prognosis. This finding may be clinically important in the evaluation of patients. We are evaluating this in more detail.

\section{Hospitalisation and mortality}

The present management programme did not obtain improved readmission or survival rates. The main purpose of most management programmes is to reduce the frequent and costly hospitalisations of patients with heart failure. Indeed, a greatly reduced readmission rate or prolonged time to first readmission has been reported in several studies. $^{21} 22273031$ On the other hand, no effect on hospitalisation rate and an increase in readmission rate have also been reported. ${ }^{25} 32$ In our study, hospitalisation rate and time to first readmission did not differ between study groups during 
follow up. We found both cardiac and non-cardiac readmission rates to be high, although patients with severe concomitant disease had been excluded from the study. The high non-cardiac health care consumption makes it difficult to explore the effects of our particular intervention programme directed to heart failure. Time to first readmission was much longer in our study than in another Swedish study with a similar heart failure population: 246 versus 142 days in the intervention group and 294 versus 106 days in the control groups, respectively. ${ }^{21}$ This may reflect good management of elderly patients with heart failure in primary care in our setting.

Reduced mortality with management programmes in heart failure has been reported in one study but, most often, data on mortality have been inconclusive. In our study, mortality was high, as expected, and no reduction was seen in the intervention group. ${ }^{28}$ However, despite the long observation time of more than three years, the present and other studies are too small to allow firm conclusions on the effects of management programmes on mortality. Thus, evidence suggests that management programmes seem to have small effects on morbidity and mortality, at least when the heart failure populations are examined in areas where health care programmes have been established.

\section{Summary}

In summary, the OPTIMAL study shows that a nurse based hospital outpatient clinic management programme is more effective than primary care in optimising doses of ACE inhibitors and in improving self care behaviour of older patients with heart failure. These changes did not seem to affect QoL or to influence hospitalisation rates or mortality. This may in part be due to good clinical practice in both hospital and primary care in our catchment area.

The use of a non-disease specific QoL questionnaire calls for careful interpretation of the data, in particular because readmission rates for non-cardiac disease are high. Taken together, our findings may suggest that the next important step in improving the strategy for heart failure care is to advocate implementation of generalised health care programmes in primary care. Also, it is important to identify groups who may benefit more from a hospital based management programme. Symptomatic patients with a new diagnosis, those with poor QoL, and patients in areas where no health programme is implemented should be the focus of future studies.

\section{ACKNOWLEDGEMENTS}

This study was supported by the Vårdal Foundation, the Swedish Heart and Lung Foundation, the Swedish Society of Medicine, and Karolinska Institutet. The authors are grateful to Dr Owe Schill, Ms Margret Lundström, and Ms Inger Bergbom for technical assistance.

\section{Authors' affiliations \\ M Mejhert, T Kahan, H Persson, M Edner, Section of Cardiology, Karolinska Institutet Danderyd Hospital, Stockholm, Sweden}

\section{REFERENCES}

1 Fryback DG, Dasbach EJ, Klein R, et al. The Beaver Dam health outcomes study: initial catalog of health-state quality factors. Med Decis Making 1993;13:89-102.

2 Stewart AL, Greenfield S, Hays RD, et al. Functional status and well-being of patients with chronic conditions: results from the medical outcomes study. JAMA 1989;262:907-13.
3 Mejhert $M$, Persson $\mathrm{H}$, Edner $M$, et al. Epidemiology of heart failure in Sweden: a national survey. Eur J Heart Failure 2001;3:97-103.

4 Stewart S, Maclntyre K, MacLeod MMC, et al. Trends in hospitalization for heart failure in Scotland, 1990-1996. Eur Heart J 2001;22:209-17.

5 Reijtsma JB, Mosterd A, de Craen, et al. Increase in hospital admission rates for heart failure in the Netherlands, 1980-1993. Heart 1996;76:388-92.

6 Franciosa JA, Wilen M, Ziesche S. Survival in men with severe chronic left ventricular failure due to either coronary heart disease or idiopathic dilated cardiomyopathy; Am J Cardiol 1983;2:403-10.

7 Ho KK, Pinsky JL, Kannel WB, et al. The epidemiology of heart failure: the Framingham study. J Am Coll Cardiol 1993;22(suppl):6A-13A

8 Muldoon MF, Barger SD, Flory JD, et al. What are quality of life measurements measuring? BMJ 1998;316:542-5.

9 McAlister FA, Lawson FME, Teo KK, et al. A systematic review of randomised trials of disease management programs in heart failure. Am J Med $2001 ; 110: 378-84$.

10 Kahan T, Wändell P. Risk factors in established coronary heart disease: evaluation of a secondary prevention programme. J Cardiovasc Risk 2001;8:73-80.

11 Hunt SM, McKenna SP, McEwen J, et al. A quantitative approach to perceived health status: a validation study. J Epidemiol Commun Health 1980;34:281-6.

12 Hunt SM, McKenna SP, McEwen J, et al. The Nottingham health profile: subjective health status and medical consultations. Soc Sci Med 1981;15A:221-9.

13 Wiklund I, Romanus B, Hunt S. Self assessed disability in patients with arthrosis of the hip joint: reliability of the Swedish version of the Nottingham health profile. Int Disabil Stud 1988;10:159-63.

14 Wiklund I, Herlitz J, Hjalmarson Å. Quality of life five years after myocardial infarction. Eur Heart J 1989; 10:464-72.

15 Anon. American Society of Echocardiography Committee on standards. Recommendations for quantification of the left ventricle by two-dimensional echocardiography. J Am Soc Echocardiogr 1989;2:358-67.

16 Höglund C, Alam M, Thorstrand C. Atrioventricular valve plane displacement in healthy persons: an echocardiographic study. Acta Med Scand 1988;224:557-62.

17 Del Ry S, Clerico A, Giannessi D, et al. Measurement of brain natriuretic peptide in plasma samples and cardiac tissue extracts by means of an IRMA method. Scand J Clin Lab Invest 2000;60:81-90.

18 Hunt SM, McEwen J, McKenna SP. Perceived health: age and sex norms in a community. J Epidemiol Commun Health 1984;38:156-60.

19 Massie BM, Armstrong PW, Cleland JGF, et al. High doses of angiotensin converting enzyme inhibitors are well tolerated in patients with chronic heart failure: results from the ATLAS trial. Arch Intern Med 2001;161:165-71.

20 Rydell-Karlsson M, Billing E, Edner M. Den sjuksköterskebaserade hiärtsviktmottagningen förbättrar patienternas kunskap. OPTIMALstudien. Hygiea $2001 ; 110: 221$.

21 Cline CMJ, Israelsson BYA, Willenheimer RB, et al. Cost effective management programme for heart failure reduces hospitalisation. Heart 1998;80:442-6.

22 Rich MW, Beckham V, Wittenberg A, et al. A multidisciplinary intervention to prevent the readmission of elderly patients with congestive heart failure. N Engl J Med 1995;333:1190-5.

23 Anon. National Corporation of Swedish Pharmacies, Stockholm, Sweden. Svensk Läkemedelsstatistik 1997:229-30.

24 Mejhert M, Holmgren J, Wändell, et al. Diagnostic tests, treatment and follow up in heart failure patients: is there a gender bias in the coherence to guidelines? Eur J Heart Failure 1999; 1:407-10.

25 Ekman I, Andersson B, Ehnfors M, et al. Feasibility of a nurse monitored, out patient care programme for elderly patients with moderate to severe, chronic heart failure. Eur Heart J 1998; 19:1254-60.

26 Strömberg A, Mårtensson J, Fridlund B, et al. Nurse led heart failure clinics in Sweden. Eur J Heart Failure $2001 ; 3: 139-44$.

27 Doughty RN, Wright SP, Pearl A, et al. Randomised, controlled trial of integrated heart failure management: the Auckland heart failure management study. Eur Heart J 2002;23:139-46.

28 Stewart S, Pearson S, Luke CG, et al. Effects of home-based intervention on unplanned readmissions and out of hospital deaths. J Am Geriatr Soc 1998:46:174-80.

29 Berry C, McMurray J. A review of quality of life evaluations in patients with congestive heart failure. Pharmacoeconomics 1999;16:247-71.

30 Krumholz HM, Amatruda J, Smith GL, et al. Randomised trial of an education and support intervention to prevent readmission of patients with heart failure. J Am Coll Cardiol 2002;39:83-9.

31 Blue L, Lang E, McMurray JJ, et al. Randomised controlled trial of specialist nurse intervention in heart failure. BMJ 2001;29:715-8.

32 Weinberger $M$, Oddone E Z, Henderson WG, for the Veterans Affairs Cooperative Study Group on primary care and hospital readmission. Does increased access to primary care reduce hospital readmissions? N Engl J Med 1996;334:1441-7. 